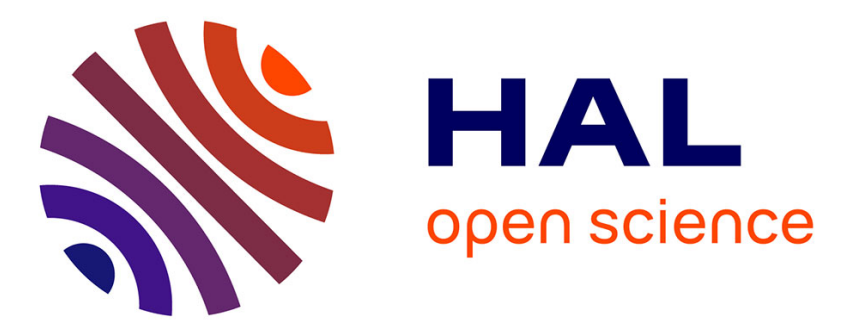

\title{
Diffusion quasiélastique de la lumière sous champ électrique : mobilité électrophorétique et charge apparente des macromolécules
}

\author{
M. Drifford, R. Menez, P. Tivant, P. Nectoux, J.P. Dalbiez
}

\section{To cite this version:}

M. Drifford, R. Menez, P. Tivant, P. Nectoux, J.P. Dalbiez. Diffusion quasiélastique de la lumière sous champ électrique: mobilité électrophorétique et charge apparente des macromolécules. Revue de Physique Appliquée, 1981, 16 (2), pp.19-33. 10.1051/rphysap:0198100160201900 jpa-00244895

\section{HAL Id: jpa-00244895 https://hal.science/jpa-00244895}

Submitted on 1 Jan 1981

HAL is a multi-disciplinary open access archive for the deposit and dissemination of scientific research documents, whether they are published or not. The documents may come from teaching and research institutions in France or abroad, or from public or private research centers.
L'archive ouverte pluridisciplinaire HAL, est destinée au dépôt et à la diffusion de documents scientifiques de niveau recherche, publiés ou non, émanant des établissements d'enseignement et de recherche français ou étrangers, des laboratoires publics ou privés. 


\title{
Diffusion quasiélastique de la lumière sous champ électrique : mobilité électrophorétique et charge apparente des macromolécules
}

\author{
M. Drifford, R. Menez, P. Tivant, P. Nectoux et J. P. Dalbiez \\ Département de Physico-Chimie, Centre d'Etudes Nucléaires de Saclay, 91190 Gif/Yvette, France
}

(Reçu le 15 juillet 1980, révisé le 15 octobre 1980, acceptéle 22 octobre 1980)

\begin{abstract}
Résumé. - La diffusion quasiélastique de la lumière sous champ électrique (DQLCE) est présentée pour permettre de déterminer les propriétés de transport de différentes macromolécules chargées. La mobilité électrophorétique des macroions est déduite du déplacement Doppler de la raie Rayleigh. La charge apparente des polyions est calculée à partir de la loi de Nernst-Einstein connaissant leur coefficient de diffusion translationnel. Cette grandeur est caractéristique des interactions coulombiennes au sein de la solution et permet d'étudier les phénomènes de condensation et de substitution de contre-ions de valences différentes.

Dans une première partie, nous donnons le principe de la DQLCE, et précisons les notions de force ionique et de charge apparente reliées à la mobilité électrophorétique. Nous décrivons ensuite l'appareillage en insistant sur les spécificités de la cellule électrophorétique et du générateur séquentiel de tension.

Les macromolécules étudiées sont alors caractérisées : l'albumine de sérum bovin (BSA), l'acide désoxyribonucléique $(\mathrm{ADN})$; le sulfate de chondroïtine $\left(\mathrm{ChSO}_{4} \mathrm{Na}\right)$; et deux polyélectrolytes synthétiques : l'acide polyméthacrylique (PMA) et un copolymère d'acide maléique et d'éthyl vinyl éther.

Enfin nous discutons de l'évolution de leur mobilité en fonction des conditions du milieu (concentration en électrolyte, concentration et masse des polyions, $\mathrm{pH}$ de la solution).
\end{abstract}

\begin{abstract}
Quasielastic light scattering in an electric field (DQLCE) is employed to determine the transport properties of several charged macromolecules. The electrophoretic mobility of the macroions is derived from the Doppler shift of the Rayleigh line. The apparent charge of the polyions is given by the Nernst-Einstein relation when the translational diffusion coefficient is known. This quantity characterizes the Coulomb interactions and enables the condensation and substitution phenomena to be studied for counter-ions of various valencies.

In a first part, we give the main principles of DQLCE and we define the concepts of ionic strength and apparent charge related to the electrophoretic mobility. Then we describe the apparatus and particularly the specific features of the electrophoretic cell and voltage timing device.

The characteristics of the macromolecules we have studied are given next : bovin serum albumin (BSA); deoxyribonucleic acid (DNA); chondroitin sulfate $\left(\mathrm{ChSO}_{4} \mathrm{Na}\right)$ and two synthetized polyelectrolytes : polymethacrylic acid (PMA) and a copolymer of maleic acid and ethyl vinyl ether.

The variations of their mobility with the conditions existing in the medium (concentration of the electrolyte, mass and concentration of the polyions, $\mathrm{pH}$ of the solution) are discussed.
\end{abstract}

1. Introduction. - La diffusion quasiélastique de la lumière émise par un laser est une technique très utilisée pour étudier les propriétés hydrodynamiques des macromolécules en solution. En effet, le profil du spectre Rayleigh reflète la dynamique de la solution. La lumière diffusée par des macromolécules animées d'un mouvement brownien présente un spectre lorentzien élargi par effet Doppler, centré sur la fréquence incidente. Son analyse en fréquence permet de déterminer les coefficients de diffusion translationnelle et rotationnelle des particules. De plus le spectre diffusé par des macromolécules soumises à un mouvement directif est déplacé en fréquence, proportionnellement à leur vitesse.
Yeh et Cummins [1] ont les premiers mis en évidence un déplacement en fréquence de la lumière diffusée par une solution de macromolécules soumises à un flux uniforme. B. R. Ware et W. H. Flygare en 1971 [2] ont proposé un couplage des techniques d'électrophorèse et de diffusion de la lumière : les macromolécules chargées migrent sous l'effet d'un champ électrique perpendiculaire au faisceau laser. Le spectre de la lumière diffusée est déplacé proportionnellement à la mobilité électrophorétique des diffuseurs.

Cette technique de diffusion quasiélastique de la lumière sous champ électrique (DQLCE) nécessite une détection hétérodyne par battement de photons 
pour mesurer des déplacements en fréquence de quelques hertz. Son principe consiste à envoyer sur le détecteur, la lumière diffusée à la fréquence $v_{\mathrm{D}}$, et une partie du faisceau incident non perturbé (oscillateur local) à la fréquence $v_{0}$.

La collecte simultanée des deux faisceaux sur la photocathode du détecteur, engendre un battement du photocourant à la fréquence $\left(v_{\mathrm{D}}-v_{0}\right)$. L'analyse de Fourier des fluctuations de ce photocourant produit un spectre diffusé lorentzien centré à la fréquence $v_{D}$ et permet ainsi une mesure de la mobilité électrophorétique $(U)$.

Deux équipes américaines, d'une part B. R. Ware à Harvard University Cambridge, et d'autre part E. E. Uzgiris à General Electric Corporation Research and Development, Schenectady, ont étudié et développé cet appareillage et ont donné de nombreuses applications orientées principalement vers le domaine médical. Citons les études sur les protéines et les macromolécules (BSA [2, 3], ADN [4], hémoglobine [5], et des applications spécifiques en recherche médicale sur les cellules sanguines [6], les bactéries [7] et les vésicules [8]). Les détails de l'instrumentation, ainsi que diverses autres applications sont donnés dans les références suivantes [9-13].

Après quelques rappels de la DQLCE nous décrivons les techniques expérimentales en insistant sur les parties importantes de l'appareillage : la cellule électrophorétique et le générateur de champ électrique. Ensuite nous développons l'application de cette technique à la détermination de la mobilité électrophorétique de différentes macromolécules (BSA, ADN, polyélectrolytes naturel et synthétiques).

L'intérêt physico-chimique de notre étude est de suivre l'évolution des mobilités en fonction de la force ionique et du $\mathrm{pH}$ du milieu, de la concentration et de la masse des polyions, et du degré d'ionisation des macromolécules. Des lois de variation expérimentales sont déduites et comparées aux théories électrostatiques des solutions de polyélectrolytes.

2. Principes et rappels théoriques. -2.1 Notion D'ÉLECTROPHORÈSE [14]. - Une macromolécule chargée soumise à un champ électrique peut migrer vers une électrode de charge opposée et acquérir une vitesse proportionnelle au champ appliqué. La constante de proportionnalité est appelée mobilité électrophorétique :

$$
\mathbf{V}=U \cdot \mathbf{E}
$$

La mobilité électrophorétique est caractéristique de la macromolécule, sa valeur dépend des conditions du milieu et peut se mettre sous la forme

$$
U=\frac{Z e}{\zeta} F(\varkappa R)
$$

$Z$ est le nombre de sites'chargés du polyion,

$e$ est la valeur de la charge élémentaire,

$\zeta$ est le coefficient de friction hydrodynamique du macroion,
$F(\varkappa R)$ est une fonction caractéristique de la forme du diffuseur et des propriétés électrostatiques de la solution,

$\varkappa$ est la constante de Debye-Hückel, proportionnelle à la racine carrée de la force ionique de la solution, soit

$$
\frac{1}{\varkappa}=\left[\frac{\varepsilon k T}{4 \pi e^{2} \sum_{i} n_{i} Z_{i}^{2}}\right]^{1 / 2}
$$

qui a la dimension d'une longueur (longueur de Debye) et représente le rayon de l'atmosphère ionique.

$\sum_{i} n_{i} Z_{i}^{2}$ est le double de la force ionique de Lewis où $n_{i}$ est la concentration molaire de chaque espèce ionique de valence $Z_{i}$.

$\varepsilon$ est la constante diélectrique du milieu et $k T$ le facteur de Boltzmann.

Pour bien définir la longueur de Debye nous devons d'abord caractériser les solutions de polyélectrolytes qui sont des macromolécules à forte densité de charge portant de nombreux groupes ionisables. Les solutions étudiées sont alors composées de macroions polyvalents et d'un grand nombre de petits ions de charge opposée (contre-ions). Elles peuvent également contenir des électrolytes ajoutés comportant des contre-ions et des co-ions.

Deux cas sont alors à considérer pour définir la force ionique :

- Une solution à deux composants ioniques : le polyion de concentration $C(g / l)$, de masse $M$, de charge $Z$ (en unité de charges élémentaires) et ses contre-ions de valence $Z_{2}$.

La force ionique de la solution est directement reliée à la concentration en contre-ions, soit :

$$
I=\frac{1}{2} \frac{C|Z|\left|Z_{2}\right|}{M} \text { en } \mathrm{M} / 1 \text {. }
$$

En considérant des solutions de macromolécules dont le contre-ion est généralement l'ion $\mathrm{Na}^{+}$, nous obtenons :

$$
I=\frac{1}{2} \frac{C|Z|}{M}
$$

Pour une solution aqueuse diluée de polyélectrolyte, l'inverse de la longueur de Debye peut alors s'écrire :

$$
\varkappa_{0}\left(\mathrm{~cm}^{-1}\right)=3,3 \times 10^{7} \sqrt{\frac{C|Z|}{2 M}} \text { à } 20^{\circ} \mathrm{C}
$$

l'indice 0 indiquera dans nos expressions, l'absence d'électrolyte ajouté.

- Une solution à trois composants ioniques : le polyion, ses contre-ions, et un sel de concentration $C_{\mathrm{s}}$, dont le contre-ion est le même que celui du polyion. 
La force ionique est de la forme :

$$
I=\frac{1}{2}\left|Z_{2}\right|\left[C_{\mathrm{s}}\left(1+\left|Z_{2}\right|\right)+\frac{C|Z|}{M}\right] .
$$

Avec du chlorure de sodium ajouté :

et

$$
I=\frac{1}{2}\left(\frac{C|Z|}{M}+2 C_{\mathrm{s}}\right)
$$

$$
x=3,3 \times 10^{7} \sqrt{\frac{C|Z|}{2 M}+C_{\mathrm{s}}} .
$$

Dans le cas de macromolécules de forme sphérique, la mobilité électrophorétique s'exprime sous une forme réduite [15] :

$$
U=\frac{Z e}{6 \pi \eta R} \frac{f(x R)}{1+x R}
$$

$\eta$ est la viscosité du milieu,

$R$ est le rayon hydrodynamique du polyion,

$f(x R)$ est la fonction de Henry [15]. Pour des macromolécules sphériques, $f(x R)$ vaut 1 quand $x R \ll 1$ et varie de 1 à 1,5 pour $x R \gg 1$.

En considérant des diffuseurs cylindriques qui peuvent représenter des polyélectrolytes linéaires rigides, la mobilité électrophorétique peut s'exprimer suivant la formule donnée par Gorin et al. [16] :

$$
U=\frac{2 Z e}{\pi \eta l} \times \frac{1}{F(\varkappa b)}\left(\frac{K_{0}(\varkappa b)}{\varkappa b \cdot K_{1}(\varkappa b)}+\ln \frac{b+r_{i}}{b}\right)
$$

$l$ et $b$ représentent la longueur et le rayon du cylindre, $K_{0}$ et $K_{1}$ sont les fonctions de Bessel d'ordre 0 et 1 , $r_{i}$ est le rayon d'hydratation de la molécule d'eau $(1,5 \AA)$,

$F(x b)$ est le facteur correctif de Henry, variant de 8 à 6,1 lorsque $x b$ varie de 0 à 3 .

Ainsi la mobilité électrophorétique doit ..varier avec le paramètre $x$ de Debye qui caractérise le potentiel électrostatique de la solution. La mobilité sera très sensible à la force ionique telle que nous l'avons définie, à la concentration du polyion et à ses caractéristiques structurales (forme, taille, et surtout charge).

2.2 Charge aPPaRente Des POlyéleCtrolytes. $\mathrm{La}$ densité de charge du polyion peu induire de fortes interactions électrostatiques avec les contre-ions dont une partie se fixe au voisinage immédiat de ses sites chargés. Ce phénomène, généralement appelé condensation ionique est prédominant dans les interactions ions-polyions; en particulier leur mobilité électrophorétique est modifiée par ce phénomène. La mobilité étant reliée à la charge, nous pouvons définir ainsi une charge apparente du polyion qui tient compte de ces intéractions.

A partir des deux paramètres déterminés expéri- mentalement par diffusion de la lumière : $D_{\mathbf{t}}$ et $U$, nous pouvons définir une charge apparente telle que :

$$
Z_{\text {app }}=\frac{U}{D_{\mathrm{t}}} \times \frac{k T}{e}
$$

$D_{\mathrm{t}}$ étant le coefficient de diffusion translationnelle du polyion.

Ce concept est tiré de relation de Nernst-Einstein [17]

$$
\frac{U_{0}}{D_{0}}=Z \frac{e}{k T}
$$

établie pour des électrolytes simples à dilution infinie, $Z$ représentant la charge structurale de l'ion.

Ainsi nous pouvons déduire de nos expériences, l'évolution de la charge apparente avec les conditions du milieu et étudier le phénomène de condensation et de substitution de contre-ions de valences différentes [18-19].

2.3 SPECTRE DE LA LUMière DIFFUSÉE SOUS CHAMP ÉLECTRIQUE. - Considérons un ensemble de macromolécules qui se déplacent dans une direction donnée et à la même vitesse sous l'influence du champ électrique. La différence de fréquence $\Delta v\left(v_{\mathrm{D}}-v_{0}\right)$ est reliée à la fréquence incidente $v_{0}$ par la formule de l'effet Doppler :

$$
\Delta v(\mathrm{~Hz})=v_{0} \cdot \frac{2 V}{c} \cos \alpha \cdot \sin \frac{\theta}{2}
$$

avec

$\theta:$ angle de diffusion,

$\alpha$ : angle entre la bissectrice extérieure de $\theta$ et la direction de la vitesse,

$c$ : vitesse de la lumière.

Remarquons que la diffusion de la lumière est due à des fluctuations locales et spontanées de la constante diélectrique du milieu. Celles-ci ayant une périodicité spatiale, les conditions de diffusion sont données par la loi de Bragg qui relie le vecteur d'onde $\mathbf{K}$ d'une fluctuation aux vecteurs d'onde $\mathbf{k}_{\mathrm{o}}$ et $\mathbf{k}_{\mathrm{d}}$ des ondes lumineuses incidente et diffusée.

Cette relation peut s'écrire :

$$
\mathbf{k}_{0}-\mathbf{k}_{\mathrm{d}}=\mathbf{K} \quad \text { où } \quad|\mathbf{K}|=\frac{4 \pi n}{\lambda_{0}} \sin \frac{\theta}{2}
$$

$\lambda_{0}$ longueur d'onde incidente,

$n$ indice moyen de réfraction de la solution.

La lumière diffusée à un angle $\theta$ provient uniquement de la fluctuation de vecteur d'onde $\mathbf{K}$, ou correspond encore à la lumière réfléchie par la composante de Fourier de vecteur $\mathbf{K}$ des fluctuations de concentration.

Exprimons le déplacement Doppler dans le cadre de l'expérience de DQLCE, soit :

$$
\Delta \omega(\mathrm{rad} . / \mathrm{s})=\mathbf{K} . \mathbf{V} \text {. }
$$


Si la direction du champ électrique est perpendiculaire au faisceau incident :

$$
\begin{aligned}
& \Delta \omega(\mathrm{rad} . / \mathrm{s})=|\mathbf{K}||\mathbf{V}| \cos \frac{\theta}{2} \\
& \Delta v(\mathrm{~Hz})=\frac{n U E}{\lambda_{0}} \sin \theta .
\end{aligned}
$$

Les théories de $\mathrm{DQL}$ ont été amplement développées dans l'étude des mélanges critiques et des phénomènes de transport des macromolécules biologiques $[20,21]$; celles-ci sont directement applicables en tenant compte de l'influence du champ électrique [9].

Les équations de base de la fonction d'autocorrélation des fluctuations de concentration, et de la densité spectrale d'un système de $N$ particules identiques et isotropes sont les suivantes :

- Sans champ électrique appliqué :

$$
C(\mathbf{K}, t)=N|A|^{2} \mathrm{e}^{-i \omega_{0} t} \cdot \mathrm{e}^{-D_{t} K^{2} t}
$$

où $A$ est l'amplitude moyenne du champ diffusé

$$
S(\mathbf{K}, \omega)=N|A|^{2} \frac{D_{\mathrm{t}} K^{2} / \pi}{\left(\omega-\omega_{0}\right)^{2}+\left(D_{\mathrm{t}} K^{2}\right)^{2}} .
$$

- Sous l'influence du champ électrique externe (Fig. 1) :

$$
\begin{gathered}
C(\mathbf{K}, t)=N|A|^{2} \mathrm{e}^{-i \omega_{0} t} \cdot \mathrm{e}^{-D_{\mathrm{t}} \mathbf{K}^{2} t} \cdot \mathrm{e}^{ \pm i \mathbf{K} . \mathbf{v} t} \\
S(\mathbf{K}, \omega)=N|A|^{2} \frac{D_{\mathrm{t}} K^{2} / \pi}{\left(\omega-\omega_{0} \pm K V \cos \frac{\theta}{2}\right)^{2}+\left(D_{\mathrm{t}} K^{2}\right)^{2}}
\end{gathered}
$$

Ainsi, dans le cas idéal d'une expérience de diffusion, les deux paramètres de transport $D_{\mathrm{t}}$ et $U$ peuvent être déduits. On détermine ainsi à partir de l'équation (12) la charge apparente du diffuseur.

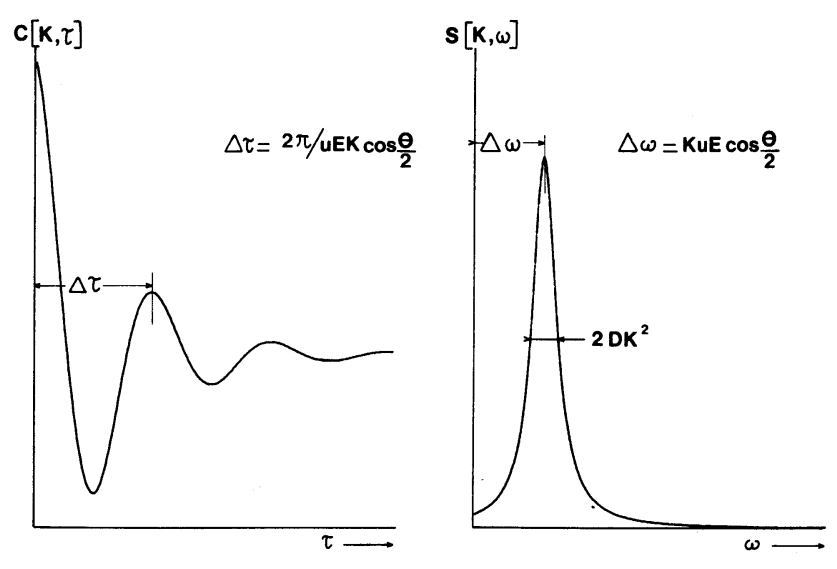

Fig. 1. - Fonction d'autocorrélation et densité spectrale de la lumière diffusée par une solution de macromolécules chargées en présence d'un champ électrique.

[Autocorrelation function and spectral density of light scattered from a solution of charged macromolecules in the presence of an electric field.]
La résolution spectrale peut alors s'écrire

$$
r=\frac{\Delta \omega}{\Gamma}
$$

où

$\Gamma=D_{\mathrm{t}} K^{2}$ demi-largeur à mi-hauteur de la lorentzienne

soit

$$
r=\frac{U E}{D_{\mathrm{t}}} \cdot \frac{\lambda_{0}}{4 \pi n} \cdot \frac{1}{\tan (\theta / 2)}
$$

La détection hétérodyne à très petits angles permet de simplifier cette expression :

$$
r=\frac{U E}{D_{\mathrm{t}}} \cdot \frac{\lambda_{0}}{2 \pi n \theta} .
$$

La résolution est d'autant meilleure que la valeur du champ électrique est élevée et que l'angle d'observation est petit.

Cette dernière relation peut encore se mettre sous la forme :

$$
r=\frac{Z_{\text {app }} e}{k T} \cdot \frac{E \lambda_{0}}{2 \pi n \theta} .
$$

La résolution dépendra donc directement de la charge apparente de la macromolécule.

3. Appareillage [22]. - Pour obtenir une résolution convenable des spectres déplacés par l'action du champ électrique sur les macromolécules et, de plus, pour avoir une valeur de l'intensité des fluctuations du photocourant exploitable pour des solutions de très faibles forces ioniques, le montage nécessite une détection hétérodyne aux très petits angles.

Le schéma de principe de l'installation dont les principales composantes seront caractérisées ultérieurement est représenté sur la figure 2 .

3.1 LASER ET OPTIQUE. - La source est constituée par un laser Hélium Néon Spectra Physics SP125 délivrant $80 \mathrm{~mW}$ à la longueur d'onde de $6328 \AA$. Il est monomode transverse $\left(\mathrm{TEM}_{00}\right)$. La largeur instantanée d'un mode longitudinal est de l'ordre du hertz. Le faisceau laser est fọcalisé sur l'échantillon par une lentille de focale $F=300 \mathrm{~mm}$. Le diamètre du faisceau laser dans le plan focal de la lentille est de $0,23 \mathrm{~mm}$. Un diaphragme $D_{1}$ permet d'éviter les réflexions multiples du faisceau. L'ensemble photomultiplicateur-diaphragmes $\mathrm{D}_{2} \mathrm{D}_{3}$ est monté sur un bras pouvant pivoter autour d'un axe vertical passant au centre du volume diffusant. Le diaphragme $\mathrm{D}_{2}$ de $1 \mathrm{~mm}$ de diamètre est placé à $200 \mathrm{~mm}$ de la cuve et la distance $D_{2} D_{3}$ est également de $200 \mathrm{~mm}$. Le photomultiplicateur est placé contre le diaphragme $\mathrm{D}_{3}$ de diamètre $1 \mathrm{~mm}$. La zone diffusante vue par le photomultiplicateur est inférieure à la zone délimitée par le diaphragme $D_{2}$, celui-ci limite simplement 


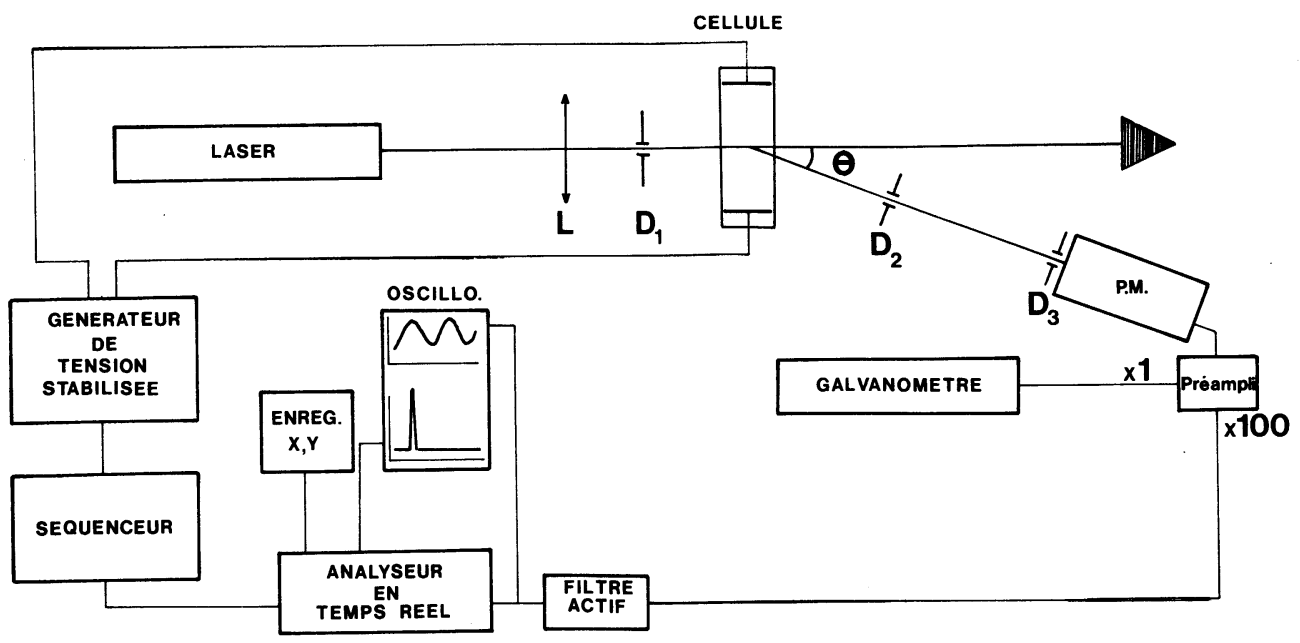

Fig. 2. - Schéma du dispositif expérimental.

[A schematic diagram of an electrophoretic light scattering apparatus.]

l'entrée de lumière parasite. L'angle apparent d'observation peut varier de $1^{\circ}$ à $12^{\circ}$ avec une précision de $\pm 6^{\prime}$. L'aire de cohérence dans laquelle le champ diffusé est spatialement cohérent décroît avec l'angle d'observation selon l'expression :

$$
A_{\mathrm{coh}}=\frac{\lambda_{0}^{2}}{\Omega}=\frac{B^{2} \lambda_{0}^{2}}{d(L \sin \theta+d|\cos \theta|)}
$$

$\Omega$ : angle solide sous lequel le photomultiplicateur voit le volume diffusant,

$d$ : diamètre du volume diffusant,

$L$ : longueur du volume diffusant : $3 \mathrm{~mm}$ (épaisseur de la cuve d'électrophorèse),

$B$ : distance de la zone diffusante au photomultiplicateur : $400 \mathrm{~mm}$,

$\theta$ : angle réel d'observation.

La surface éclairée de la photocathode du photomultiplicateur est délimitée par le diaphragme $\mathrm{D}_{3}$ : surface de $D_{3}=0,78 \mathrm{~mm}^{2}$. Le nombre d'aires de cohérence collectées par le PM décroît avec l'angle d'observation. Par exemple, à $5^{\circ}$ apparent $(\theta$ réel : $3,75^{\circ}$ ), qui est l'angle utilisé pour notre étude, le photomultiplicateur voit environ 1 aire de cohérence. L'intensité du photocourant, est alors diminuée par le facteur $\left(A_{\mathrm{coh}} / A_{\mathrm{PM}}\right)^{1 / 2}$, où $A_{\mathrm{PM}}$ est l'aire du photomultiplicateur.

Le laser, l'optique, la cellule électrophorétique et le photomultiplicateur sont montés sur un système antivibratoire (bacs de sable, marbre sur vibrachocs à coussins d'air) pour éliminer toutes les vibrations mécaniques de basses fréquences.

3. 2 Cellule électrophorétique. - La géométrie idéale de la cellule contenant les macromolécules à étudier est difficile à définir. En effet, il faut tenir compte de plusieurs critères, en particulier pour minimiser les perturbations entraînées par le passage du courant électrique.
Les caractéristiques principales de la cellule doivent être soumises aux conditions suivantes :

- uniformité du champ dans le volume diffusant,

- faibles perturbations aux électrodes,

- minimisation des phénomènes d'électroosmose et d'effet Joule (convection) dans la solution,

- volume diffusant peu important, essentiellement pour les produits biologiques,

- manipulation et démontage de la cellule le plus simple possible.

Nous avons utilisé une cellule simple dont le volume n'est pas négligeable $\left(\sim 5 \mathrm{~cm}^{3}\right)$, mais pour laquelle les phénomènes d'électroosmose et de convection sont relativement faibles.

Cette cellule est en forme de U [23] (Fig. 3) et comprend deux parties distinctes :

- une partie optique (A) de forme parallélépipédique de $20 \times 5 \times 3 \mathrm{~mm}^{3}\left(V=0,3 \mathrm{~cm}^{3}\right)$,

- une partie réservoir composée des deux branches $B$ et $\mathbf{C}$ cylindriques que l'on peut éventuellement

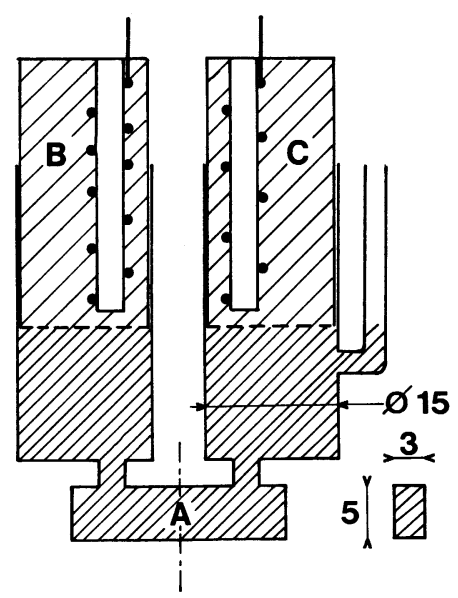

Fig. 3. - Coupe de la cellule électrophorétique.

[Cross-section of the electrophoretic light scattering cell.] 
isoler de la partie A par une membrane de dialyse, évitant ainsi le contact des électrodes avec les macromolécules.

Les électrodes sont des fils de platine enroulés autour d'une tige de verre.

3.2.1 Phénomènes thermiques. - L'effet Joule perturbe considérablement la résolution et la reproductibilité du déplacement Doppler, car il introduit des convections et des instabilités du type Bénard qui dépendent de la géométrie de la cellule. Nous avons adopté les techniques opératoires de Ware [9] en séparant les impulsions du champ électrique par des temps de repos assez longs. Ainsi, entre deux mesures, l'effet Joule produit par le passage du courant est dissipé par un échange thermique avec l'extérieur. De manière générale, il est très difficile d'étudier des solutions de force ionique élevée dans lesquelles la conductivité est importante. Nos expériences nécessitent donc un compromis entre le temps de passage du courant et la valeur du champ appliqué.

Evaluons l'échauffement provoqué par l'effet Joule pour une expérience typique, avec les paramètres suivants : $I=1 \mathrm{~mA}$ pour une application d'une tension de $100 \mathrm{~V}$ pendant un temps de $10 \mathrm{~s}$ (distance entre les électrodes $\simeq 6 \mathrm{~cm}$ et section de la cellule $\sim 0,2 \mathrm{~cm}^{2}$ ), pour une solution de conductivité spécifique de $2 \times 10^{-3} \Omega^{-1} \cdot \mathrm{cm}^{-1}$. L'élévation de température est de $3 \times 10^{-2}{ }^{\circ} \mathrm{C}$, donc négligeable devant les autres causes de fluctuations thermiques (température ambiante $22^{\circ} \mathrm{C}$ ).

3.2.2 Phénomènes d'électroosmose. - Le contact d'une solution de macromolécules chargées avec les parois de la cellule optique peut entraîner une adsorption d'ions sur celles-ci. Sous l'application d'un champ électrique, on observe alors une distribution de vitesses caractéristiques de la distance de la particule qui diffuse à la paroi. Ce phénomène d'électroosmose perturbe donc la solution au voisinage des parois où il peut s'établir des migrations de sens opposés à celui imposé par le champ appliqué. Le déplacement Doppler étudié ne correspond alors pas à la vitesse électrophorétique réelle des macromolécules [24].

Les caractéristiques géométriques de la cellule de mesure tendent à minimiser cet effet : la section diffusante de $0,15 \mathrm{~cm}^{2}$ est très supérieure à la valeur limite (cellule du type capillaire) pour laquelle ce phénomène est observé [3]. D'autre part, la mesure. de la mobilité électrophorétique d'une macromolécule, pour différentes positions du faisceau laser par rapport aux faces horizontales de la cellule, donne des valeurs constantes à $5 \%$ près jusqu'à $1,5 \mathrm{~mm}$ des parois. La reproductibilité et la valeur absolue des mesures expérimentales du déplacement Doppler pour des macromolécules connues (BSA), sont en bon accord avec les valeurs des mobilités électrophorétiques mesurées par les techniques classiques d'électrophorèse [2]. Par conséquent, pour notre type de cellule, l'effet d'électroosmose semble négligeable pour la détermination de la valeur absolue de la mobilité.

3.2.3 Phénomènes aux électrodes. - Trois caractéristiques propres à cette cellule minimisent les phénomènes aux électrodes :

- des électrodes de platine ou de Pt platiné enroulées autour d'une tige de verre ont été utilisées de façon à ce que les bulles formées par électrolyse se dégagent rapidement et perturbent peu l'intensité du champ électrique;

- les dégagements gazeux pouvant créer des différences de pression entre les deux branches de la cuve et ainsi provoquer des perturbations hydrodynamiques dans le volume diffusant, ont été éliminés par une mise à l'air aux sommets des bouchons qui maintiennent les électrodes ;

- la séparation par une membrane de dialyse permet d'éviter le contact des électrodes avec les macromolécules et ainsi leur dénaturation. De même les décompositions électrolytiques n'atteignent pas le volume diffusant.

En conclusion, les caractéristiques d'une cellule sont conçues en fonction du problème à étudier : B. B. Ware [11] et E. E. Uzigiris [25] ont proposé différents types de cellule électrophorétique.

3.3 GÉNÉRATEUR DE SÉQuENCES. - Ce générateur est destiné d'une part à découper une haute tension continue en créneaux de période et de rapport cyclique variables, et d'autre part, à déclencher l'analyse. Les raisons qui ont motivé ses caractéristiques sont les suivantes :

\section{- Tension alternée}

Ce type de tension est impératif afin d'éviter la formation de gradient de concentration dans la cellule.

\section{- Rapport cyclique variable}

Entre le créneau positif et le créneau négatif un temps de repos est nécessaire afin que la solution retrouve son équilibre thermique. L'analyse du signal ne doit se faire, bien sûr, que pendant l'application du champ. La durée d'un créneau doit être suffisante pour permettre l'acquisition des données par l'analyseur et dépend de la gamme de fréquence explorée.

Le chronogramme du séquenceur est représenté sur la figure 4.

La période " $T$ » est le temps s'écoulant entre deux montées de créneaux positifs de tension. Si l'on désigne par. " $d$ » la durée de ces créneaux, le rapport cyclique $" H$ » a pour expression $H=d / T$. Le temps de repos entre deux créneaux dépendra du courant, de son temps de passage et de la résistance de la solution.

\section{- Retard à la mesure}

Il est intéressant de ne commencer la mesure que quelques instants après l'application de la tension 


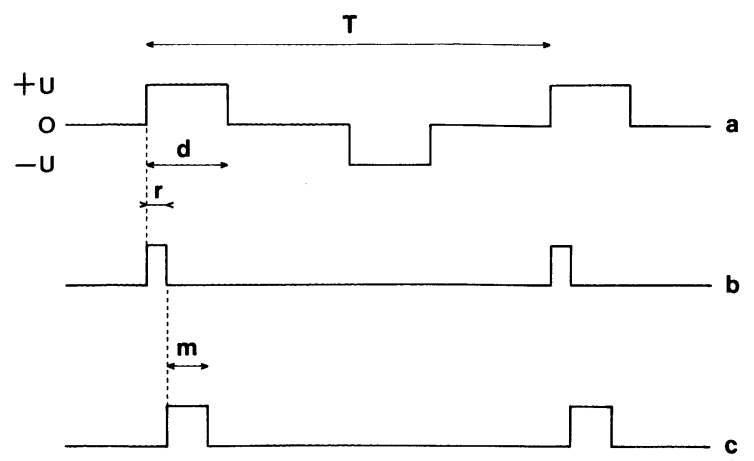

Fig. 4. - Chronogramme du séquenceur utilisé pour la tension : a) signal appliqué aux électrodes ; $b$ ) signal de retard à la mesure : c) signal de déclenchement de la mesure.

[Time diagram used for the electrical field. a) applied signal to electrodes; $b$ ) delayed signal to measure; $c$ ) pulsed signal for measure.]

afin de permettre aux macromolécules d'avoir atteint leur vitesse limite. Ce temps de retard dépend de la solution étudiée. Le temps " $m$ " représente la durée de l'analyse à chaque période, et " $r$ » le retard entre l'application de la tension et le début de la mesure.

Le choix de la période se fait par division de la fréquence d'un oscillateur à transistor unijonction. Elle peut varier de 20 à $3200 \mathrm{~s}$. Le rapport cyclique, variant de $T / 5$ à $T / 20$, est décodé au niveau du dernier compteur de la chaîne de diviseurs. Un circuit bascule permet d'alterner le sens des créneaux en conservant le rapport cyclique des impulsions. Ces signaux découpent la haute tension par l'intermédiaire de deux relais montés en inverseurs de polarité. Le déclenchement de l'analyseur est obtenu à partir de la sortie de la bascule par deux monostables montés en cascade, assurant respectivement le retard et la mesure sur les créneaux positifs. Le retard peut varier continuement de $1 / 5$ de seconde à $4 \mathrm{~s}( \pm 2 \%)$ et la mesure de $1 \mathrm{~s}$ à $40 \mathrm{~s}( \pm 3 \%)$. Un circuit de coïncidence évite que la mesure se poursuive après l'application du champ.

3.4 DéteCtion et ANALYSE. - L'oscillateur local, nécessaire à la détection hétérodyne, est fourni par une partie du faisceau laser incident, diffusée élastiquement par les irrégularités du verre de la face de sortie de la cellule optique. Celle-ci est placée sur une platine goniométrique permettant d'une part d'ajuster l'intensité de l'oscillateur local et d'autre part d'orienter les faces de la partie optique perpendiculairement au faisceau incident.

La détection est réalisée par un photomultiplicateur Hamamatsu R 374 muni d'une photocathode multi-alcaline (Cs-Na-K-Sb). L'amplification est de $5,3 \times 10^{5}$ et le bruit de $0,5 \mathrm{nA}$.

Les composantes fluctuante et continue sont amplifiées et le signal peut être éventuellement filtré afin d'éliminer les basses fréquences. L'analyse est faite par un analyseur en temps réel SAICOR 51A couplé au générateur de séquences. L'analyse instantanée du signal et le résultat de l'intégration sont visualisés sur un écran d'oscilloscope. Les spectres sont enregistrés sur une table traçante $X Y$ ou photographiés sur l'écran.

L'écart de fréquences mesuré en fonction du champ appliqué entre les électrodes varie linéairement avec celui-ci (éq. (18)). Ainsi la mobilité des macromolécules diffusantes est déduite directement de la pente de la droite $\Delta v=f(E)$.

Il est préférable, dans certains cas (force ionique élevée, forte concentration), de laisser la valeur du champ électrique constante et de faire varier l'angle d'observation.

4. Préparation et caractéristiques principales des échantillons. - L'étude des propriétés de transport des polyélectrolytes en solution aqueuse nécessite deux conditions essentielles :

- une bonne connaissance des propriétés physicochimiques de la solution : (force ionique et $\mathrm{pH}$ du milieu, concentration et masse moléculaire du soluté),

- une préparation sans corps étrangers (poussières, microprécipités), qui perturbent très fortement le phénomène de diffusion.

Une filtration des solvants sur millipore $(0,025 \mu)$ est nécessaire. On procède ensuite à une ultracentrifugation des solutions (40 $000 \mathrm{rpm}$ moyenne) ou à une filtration sur millipore $(0,22 \mu$ à $0,45 \mu)$.

Les diverses manipulations sont effectuées dans une enceinte dépoussiérée (ADS laminaire MV29), (rinçage, nettoyage des cellules, remplissage avec des seringues stériles).

Nous avons étudié la mobilité électrophorétique de diverses solutions de macromolécules biologiques ainsi que de polyélectrolytes synthétiques et naturels, qui ont été préparées dans les conditions suivantes :

- l'albumine de sérum bovin (BSA) fourni par Sochibo (Koch Light, Ref. 0142) est une protéine globulaire de masse 69000 . La solution utilisée à différentes concentrations est dialysée contre de l'eau trisdistillée pendant environ $24 \mathrm{~h}$ pour éliminer les contre-ions stoechiométriquement en excès; la charge structurale de la macromolécule est fixée par la quantité de soude ajoutée à la solution isoionique avec une mesure précise du $\mathrm{pH}$. La variation de la force ionique de la solution est obtenue en ajoutant du chlorure de sodium ;

- l'acide désoxyribonucléique (ADN) a été fourni d'une part par le Centre de Recherches des Macromolécules de Strasbourg (poids moléculaire 300000 , $\left.M_{\mathrm{w}} / M_{\mathrm{n}} \simeq 1,1\right)$ et d'autre part par Boehringer Mannheim (Réf. 15130, extrait du thymus de veau, de masse $\left.11,5 \times 10^{6}\right)$;

- le sulfate de chondroïtine de sodium $\left(\mathrm{ChSO}_{4} \mathrm{Na}\right)$ est un polysaccharide anionique de masse 14000 , le poly B-D-glycopyranosyluronique 2 desoxy-2 acetylaminogalactose sulfate. C'est le principal constituant de la substance fondamentale du cartilage et 
de la matière organique de l'os. Il en existe deux isomères principaux, différant par la position du radical sulfate. L'entité monomérique a une masse molaire de 442. La distance entre groupes ioniques est de $6 \AA$ et la longueur globale de la chaîne est d'environ $300 \AA$ [26]. Les solutions de $\mathrm{ChSO}_{4} \mathrm{Na}$ ont été préparées à partir d'un produit Sigma, isomère type $\mathrm{A}$ (Réf. C-4 134). Par passage sur une colonne de résine échangeuse de cations (Dower $50 \mathrm{WX} 8$ ) on obtient la forme acide $\mathrm{ChSO}_{4} \mathrm{H}$. La concentration ionique équivalente est déterminée par addition d'une solution titrée de soude : $1 \mathrm{~g}$ de $\mathrm{ChSO}_{4} \mathrm{Na}$ possède $3,4 \times 10^{-3}$ sites chargés, ce qui correspond à une charge structurale d'environ - 50. Le sel de calcium $\left(\mathrm{ChSO}_{4} \mathrm{Ca}\right)$ ou de tétraméthyl ammonium $\left(\mathrm{ChSO}_{4} \mathrm{TMA}\right)$ est préparé par neutralisation de la forme acide du polyion par l'hydroxyde titré correspondant ;

- l'acide polyméthacrylique (PMA) est un polyacide faible dont la formule du monomère est :<smiles>CCC(C)(C)C(=O)O</smiles>

Ces échantillons $\left(M_{\mathrm{w}}=13000\right)$ ont été fournis par M. Moan (Faculté des Sciences de Brest) et préparés par R. Zana (C.R.M. de Strasbourg). Ils sont obtenus par polymérisation en masse sous irradiation UV du monomère purifié ; ensuite le polymère est fractionné en utilisant le méthanol comme solvant et le benzène comme précipitant. Les solutions de sel de sodium du PMA dans l'eau trisdistillée sont passées sur une résine échangeuse d'ions pour obtenir la forme acide. La concentration est déterminée par dosage conductimétrique sous addition d'une solution de soude 0,1 M. Le degré de neutralisation stoechiométrique du polymère, $\alpha_{s}$, est alors obtenu par addition de soude à la solution acide $\left(\alpha_{\mathrm{s}}=1\right.$ pour l'échantillon étudié).

- Le copolymère alterné d'acide maléique et d'éthyl vinyl éther de formule générale<smiles>CCOC(CC)CCC(C(=O)O)C(C)C(=O)O</smiles>

est un polyacide synthétique.

Les échantillons ont été fournis par le Centre de Recherche des Macromolécules de Strasbourg et étudiés par J. P. Meullenet et al. [27]. Les conditions de préparation des échantillons du polymère ainsi que celles des solutions sont précisées dans la référence [28].

- Les billes de polystyrène latex proviennent de Fullam (Réf. 1050). Les billes sont calibrées à $0,109 \mu$ de diamètre et mises en suspension dans l'eau trisdistillée ou dans des solutions salines. Ce type de préparation des billes de polystyrène latex donne une dispersion colloïdale de sphères de polymère hydrophobes, stabilisées par une double couche d'ions émulsifiants anioniques adsorbés et de leurs contre-ions monovalents. Les particules portent généralement une charge négative essentiellement due à cette couche d'émulsifiant.

5. Résultats et discussion. - Deux exemples typiques montrent d'une part, un spectre déplacé sous l'influence du champ électrique $(E)$ et d'autre part, l'évolution du déplacement Doppler en fonction de $E$ (Fig. 5).
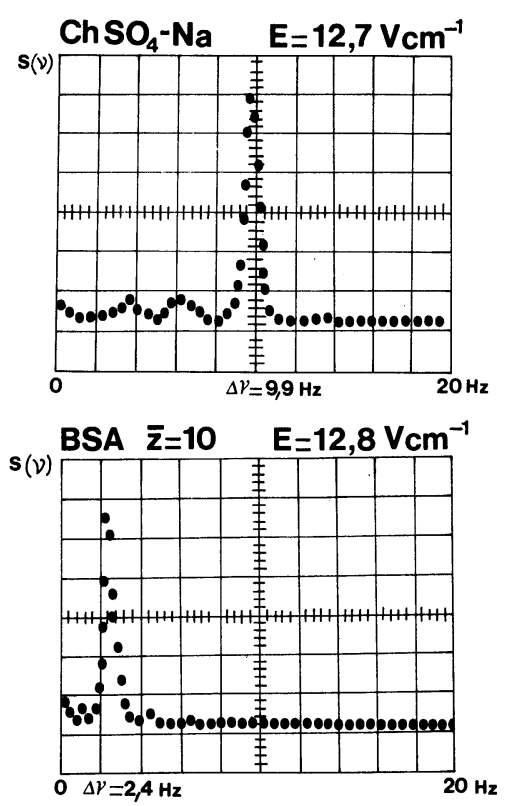

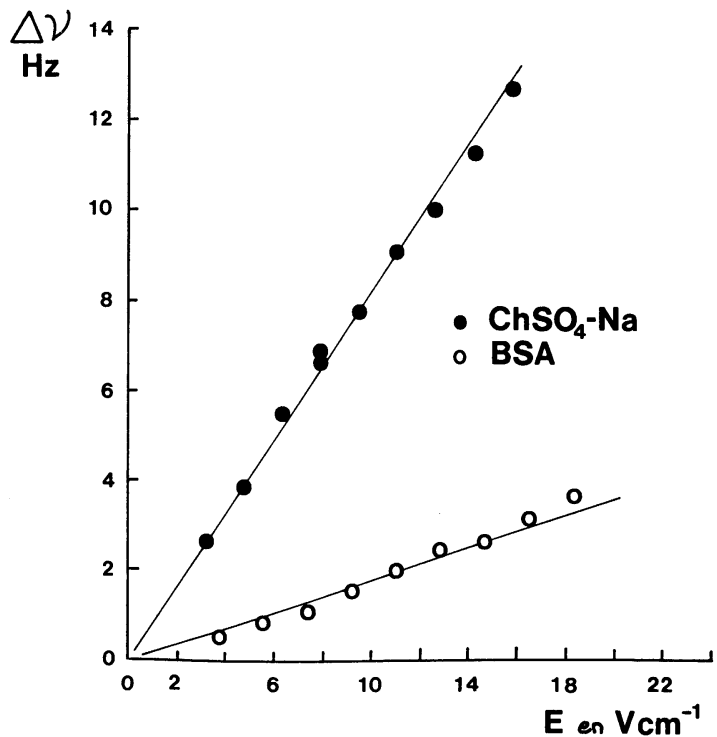

Fig. 5. - Spectres Doppler de l'albumine de sérum bovin et du sulfate de chondroïtine de sodium et évolution du déplacement en fréquence avec le champ électrique.

[Electrophoretic light scattering spectra for albumin and chondroitin sulfate and plot of the electrophoretic Doppler shift of BSA and $\mathrm{ChSO}_{4} \mathrm{Na}$ versus the electric field.] 
- L'albumine de sérum bovin (BSA) à $\mathrm{pH}=6,8$ $(Z=-10)$, sans sel ajouté et à une concentration de $50 \mathrm{~g} / 1$ a une mobilité électrophorétique à $20^{\circ} \mathrm{C}$ de $1,55 \times 10^{-4} \mathrm{~cm}^{2} \cdot \mathrm{s}^{-1} \cdot \mathrm{V}^{-1} \cdot \mathrm{A} \mathrm{pH}=9(Z=-18)$, dans les mêmes conditions de concentration, $U=3,6 \times 10^{-4} \mathrm{~cm}^{2} \cdot \mathrm{s}^{-1} \cdot \mathrm{V}^{-1}$. Ces résultats sont tout à fait comparables aux données de Ware [2] et Mohan [3].

Le sulfate de chondroitine de sodium $\left(\mathrm{ChSO}_{4}-\mathrm{Na}\right)$ en solution aqueuse à une concentration de $1,5 \mathrm{~g} / 1$ montre, d'une part, un spectre déplacé de $\Delta v=9,9 \mathrm{~Hz}$ sous un champ de $12,7 \mathrm{~V} / \mathrm{cm}$ et d'autre part, une évolution linéaire du déplacement Doppler avec le champ. La mobilité déduite est de $5,7 \times 10^{-4} \mathrm{~cm}^{2} \cdot \mathrm{s}^{-1} \cdot \mathrm{V}^{-1}$.

5.1 MOBILITÉ ÉLECTROPHORÉTIQUE DE DIFFÉRENTS SELS DE SULFATE DE CHONDROÏTINE-CHARGE APPARENTE [29]. - Les différents résultats obtenus sont illustrés sur la figure 6. Pour une même valence, la

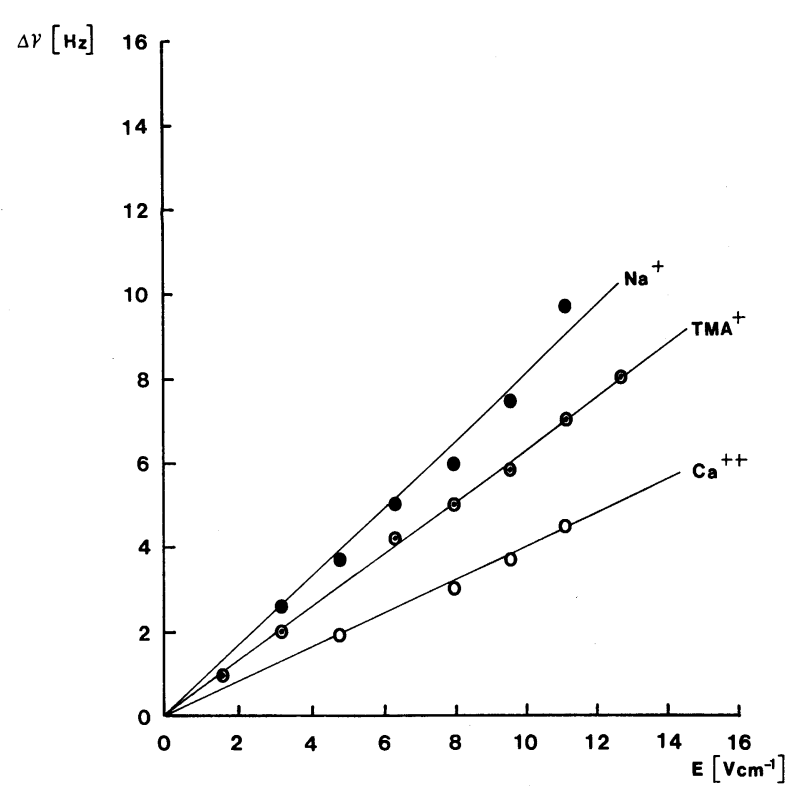

Fig. 6. - Déplacement en fréquence du spectre de la lumière diffusée par différents sels de sulfate de chondroïtine soumis à un champ électrique.

[Frequency shift for various salts of chondroitin sulfate versus electrical field.]

mobilité électrophorétique décroît quand la taille $\mathrm{du}$ contre-ion augmente $\left(\mathrm{Na}^{+}\right.$et $\left.\mathrm{TMA}^{+}\right)$. De même pour une taille équivalente, elle décroît lorsque la valence du cation augmente $\left(\mathrm{Na}^{+}, \mathrm{Ca}^{2+}\right)$. Le tableau I donne les valeurs de la mobilité électrophorétique de la macromolécule en fonction de la nature du contreion, sa charge apparente définie par la relation (12) et sa charge théorique calculée à partir du modèle de Manning qui admet la fixation d'une proportion donnée de contre-ions sur la chaîne du polyélectrolyte; cette fraction de contre-ions condensés dépend
Tableau I. - Mobilité électrophorétique et charge apparente de différents sels de sulfate de chondroïtine.

[Electrophoretic mobility and apparent charge of various salts of chondroitin sulfate.]

\begin{tabular}{|l|c|c|c|c|}
\hline $\begin{array}{c}\text { Nature du } \\
\text { contre-ion }\end{array}$ & $\begin{array}{c}U \\
\mathrm{~cm}^{2} \cdot \mathrm{s}^{-1} \mathrm{~V}^{-1}\end{array}$ & $Z_{\text {app }}=\frac{U}{D_{\mathrm{t}}} \cdot \frac{k T\left(^{*}\right)}{e}$ & $Z$ & $Z_{\text {Manning }}$ \\
\hline $\mathrm{Na}^{+}$ & $5,7 \times 10^{-4}$ & -42 & -50 & -44 \\
$\mathrm{TMA}^{+}$ & $5 \times 10^{-4}$ & -36 & -50 & -44 \\
$\mathrm{Ca}^{2+}$ & $2,8 \times 10^{-4}$ & -20 & -50 & -22 \\
$\mathrm{La}^{3+}$ & $1,9 \times 10^{-4}$ & -14 & -50 & -15 \\
\hline
\end{tabular}

$\left(^{*}\right)$ La valeur de $D_{\mathrm{t}}: 3,5 \times 10^{-7} \mathrm{~cm}^{2} \cdot \mathrm{s}^{-1}$ a été mesurée par diffusion par traceurs pour une même concentration en polyélectrolyte, et en diffusion quasiélastique en excès de sel.

essentiellement de leur valence [29]. La charge selon Manning est donnée par l'expression

$$
Z_{\text {Manning }}=Z \times \xi^{-1}\left|Z_{i}\right|^{-1}
$$

$Z$ représente la charge due à tous les groupements ionisés,

$Z_{i}$ est la valence du contre-ion,

$\xi$ est un paramètre de densité de charge; nous avons pris pour $\xi$ la valeur de 1,15 obtenue à partir de mesures thermodynamiques [30].

L'accord entre la charge apparente mesurée et la charge calculée selon Manning est convenable, pour les ions de petite taille ce qui confirme que l'approximation de Debye-Hückel, qui est la base du traitement théorique de Manning, est tout à fait valable pour ce type du polyélectrolyte peu chargé.

5.2 Charge APPaRente de L'Albumine DE SÉRUM BOVIN. - A $\mathrm{pH}=9$ et pour une concentration de $50 \mathrm{~g} / 1$, la mobilité est égale à $3,6 \times$ $10^{-4} \mathrm{~cm}^{2} \cdot \mathrm{s}^{-1} \cdot \mathrm{V}^{-1}$. Connaissant son coefficient de diffusion translationnelle : $6,5 \times 10^{-7} \mathrm{~cm}^{2} \cdot \mathrm{s}^{-1}$ déterminé en excès de sel [31,32], la charge apparente déduite par l'expression de Nernst-Einstein (éq. (12)) est égale à -14 , alors que la charge structurale est -18 . A $\mathrm{pH}=6,8$, dans les mêmes conditions, la charge apparente est de -6 alors que la charge structurale est de -10 .

A partir de la relation (10) appliquée au cas de la BSA avec $f\left(\varkappa_{0} R\right) \simeq 1$ et de l'expression de la mobilité expérimentale :

$$
U_{0}^{\exp }=\frac{Z_{\text {app }} e}{6 \pi \eta_{0} R},
$$

nous pouvons relier de manière phénoménologique la charge apparente ainsi définie, à la charge structurale du polyion et à sa concentration

$$
Z_{\mathrm{app}}=\frac{Z}{1+x_{0} R}=\frac{Z}{1+3,3 \times 10^{7} R \sqrt{\frac{C|Z|}{2 M}}} .
$$


Suivant la valeur de la charge introduite dans l'expression de la force ionique, on obtient les résultats résumés dans le tableau II. A $\mathrm{pH}=6,8$ la charge apparente calculée est en très bon accord avec la valeur expérimentale. Lorsque la protéine est plus chargée, la différence est plus accentuée.

Tableau II. - Charges structurale, apparente expérimentale, et écrantée pour la BSA à différents $p H$.

[Structural experimental apparent and screened charges for BSA at various $\mathrm{pH}$.]

$$
\begin{array}{lcccccc}
\mathrm{pH} & Z & Z_{\text {app }} & x_{0} R(Z) & Z_{\text {app }}^{1} & \varkappa_{0} R\left(Z_{\text {app }}\right) & Z_{\text {app }}^{2} \\
- & - & - & - & - & - & -11 \\
9 & -18 & -14 & 0,919 & -9 & 0,751 & -11 \\
6,8 & -10 & -6 & 0,685 & -6 & 0,531 & -6,5
\end{array}
$$

$Z_{\text {app }}^{1}$ est la charge apparente déduite de l'expression (29) en considérant

$$
x_{0} \simeq K \sqrt{\frac{C|Z|}{2 M}}
$$

$Z_{\text {app }}^{2}$ est la charge apparente obtenue par un calcul itératif en prenant :

$$
x_{0} \simeq K \sqrt{\frac{C\left|Z_{\mathrm{app}}\right|}{2 M}} .
$$

En considérant uniquement les contre-ions non condensés dans l'expression de la force ionique (tableau II, colonne 7), on obtient des valeurs calculées de la charge apparente plus conformes aux données expérimentales, surtout aux valeurs de $\mathrm{pH}$ élevées. Remarquons que cet effet est important dans le cas des macromolécules très chargées ayant un paramètre de densité de charge $\xi$ supérieur à 2 . Pour la protéine BSA, cet effet n'est pas très important puisque sa densité de charge est faible.

En présence de sel ajouté, l'expression (29) devient alors :

$$
Z_{\mathrm{app}}=\frac{Z}{1+3,3 \times 10^{7} R \sqrt{\frac{C|Z|}{2 M}+C_{\mathrm{s}}}} .
$$

Ainsi la charge apparente de la protéine décroît avec la concentration en sel ajouté, et varie théoriquement en $C_{\mathrm{s}}^{-1 / 2}$, en première approximation.

Le tableau III donne les différentes valeurs calculées de la charge apparente de la BSA à $\mathrm{pH}=6,8$ et

Tableau III. - Evolution de la charge apparente écrantée par l'effet électrophorétique en fonction de la force ionique pour la BSA.

[Evolution of apparent charge screened by electrophoretic effect versus ionic strength for BSA.]

$$
\begin{aligned}
& I=\frac{C|Z|}{2 M}+ \\
& \begin{array}{llllllllll}
+C_{\mathrm{s}}(\mathrm{mM}) & 3,5 & 4 & 6 & 10 & 20 & 50 & 100 & 1000
\end{array} \\
& \left|Z_{\text {app }}\right| \quad 5,9 \quad 5,8 \quad 5,3 \quad 4,6 \quad 3,8 \quad 2,2 \quad 2,2 \quad 0,8
\end{aligned}
$$

$C=50 \mathrm{~g} / \mathrm{l}$ en fonction de la force ionique de la solution.

Ces valeurs déduites de l'approximation de DebyeHückel en considérant l'effet électrophorétique en $(1+\chi R)^{-1}$ sont en accord avec l'estimation de la charge apparente écrantée qui justifie la décroissance $\mathrm{du}$ coefficient de diffusion translationnel apparent $D_{\text {app }}$ en fonction du sel ajouté [43].

5.3 Evolution DE LA MOBILITÉ ÉLECTROPHORÉTIQUE EN PRÉSENCE DE SEL AJOUTÉ. - Dans les solutions de polyélectrolytes, les interactions électrostatiques importantes entre polyions et contre-ions, peuvent être fortement diminuées par addition de sel. En excès de sel, les macromolécules tendent à se comporter comme des entités neutres; le coefficient de diffusion translationnelle reflète alors essentiellement le mouvement hydrodynamique des diffuseurs.

Lorsque l'on augmente de manière continue la force ionique de la solution de macromolécules par addition de sel, à concentration constante en polyions, leurs propriétés de transport sont donc modifiées. Expérimentalement la mobilité électrophorétique décroît toujours avec la quantité de sel ajouté.

Nous avons vu, d'après la relation (10) que pour des macromolécules globulaires ou des polyélectrolytes en pelote statistique, en régime dilué, la mobilité doit théoriquement varier en $C_{\mathrm{s}}^{-1 / 2}$, essentiellement si la contribution des contre-ions de la macromolécule à la force ionique, $C|Z| / 2 M$ est négligeable devant celle qui provient de l'électrolyte ajouté. La valeur de $x R$ étant souvent très inférieure à 1 et en considérant que la viscosité de la solution et le rayon globulaire de la macromolécule sont indépendants de $C_{\mathrm{s}}$, la relation (10) peut se mettre sous la forme :

$$
U \simeq U_{0}\left(1-k C_{\mathrm{s}}^{+1 / 2}\right)
$$

où

$$
U_{0}=\frac{Z e}{6 \pi \eta R} \quad \text { et } \quad k \simeq 3,3 \times 10^{7} R .
$$

On observe effectivement cette évolution expérimentale avec $C_{\mathrm{s}}$ pour les macromolécules suivantes :

- le copolymère d'acide malcique et d'éthyl vinyl ether $\left(M_{\mathrm{w}}=560000\right.$, concentration en polyion $C=0,5 \%$, degré de neutralisation $\alpha_{\mathrm{s}}=0,6$ ), pour des concentrations en $\mathrm{NaBr}$ variant de $10^{-2}$ à $10^{-1} \mathrm{M} / \mathrm{L}$ (Fig. 4 de la réf. [27]),

- le PMA $(C=1 \mathrm{~g} / \mathrm{l})$ pour des concentrations en $\mathrm{NaCl}$ ajouté comprise entre $10^{-3}$ et $10^{-1} \mathrm{M} / \mathrm{L}$ (Fig. 7),

- l'ADN [4, 22], pour les deux échantillons de masses différentes (Fig. 8), $(C=0,1 \mathrm{~g} / \mathrm{l})$ dont les mesures ont été faites pour des forces ioniques en sel ajouté variant de $10^{-3}$ à $0,15 \mathrm{M} / \mathrm{L}$. La variation est linéaire en $C_{\mathrm{s}}^{+1 / 2}$ jusqu'à $C_{\mathrm{s}}=5 \times 10^{-2} \mathrm{M} / \mathrm{L}$. Audelà de cette valeur, la mobilité décroît plus lentement et tend vers une valeur constante $[40,48]$. 


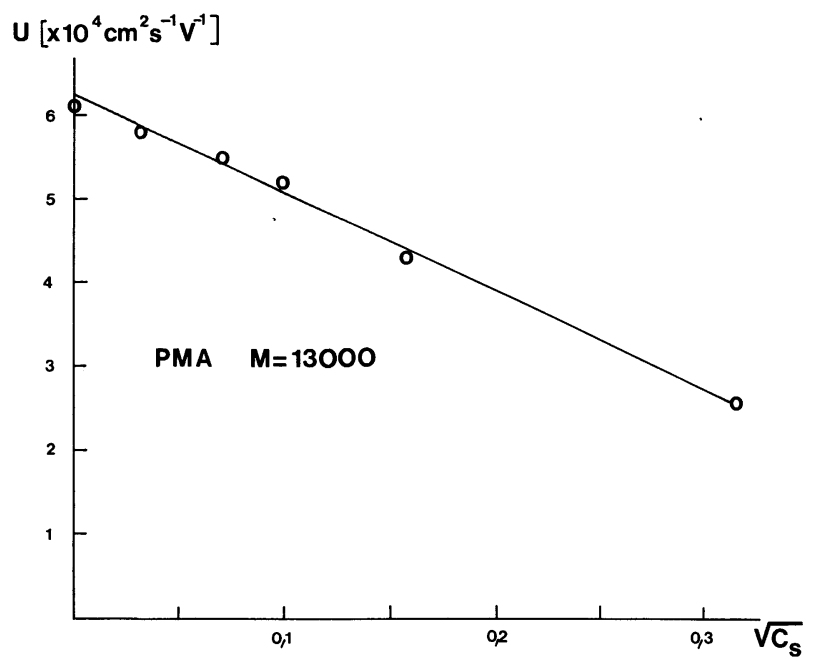

Fig. 7. - Variation de la mobilité électrophorétique du PMA avec la quantité de sel ajouté.

[Dependence of electrophoretic mobility of PMA on the square root of the $\mathrm{NaCl}$ concentration.]

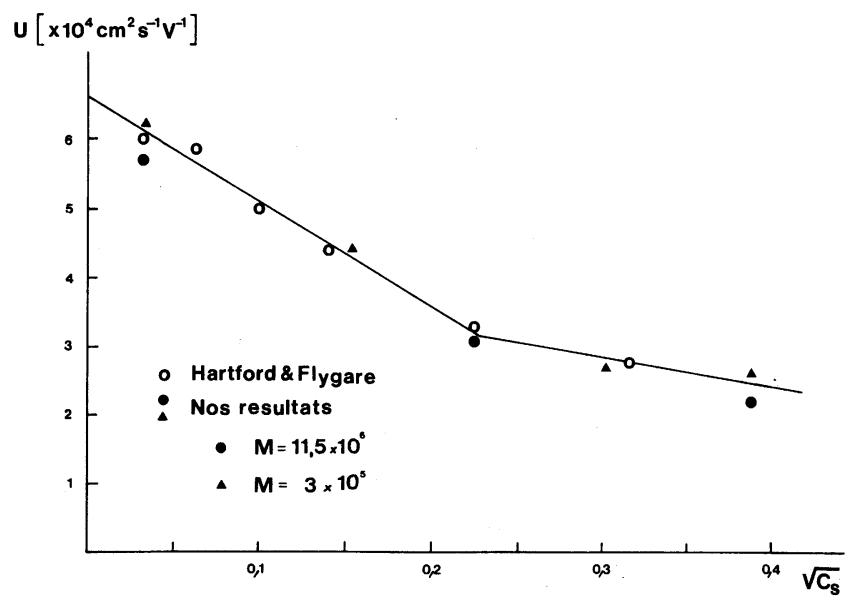

Fig. 8. - Evolution de la mobilité électrophorétique de l'ADN de différentes masses avec la quantité de sel ajouté.

[Variation of electrophoretic mobility of ADN with various molecular weights under the addition of $\mathrm{NaCl}$.]

L'extrapolation à $C_{\mathrm{s}}=0$ de la mobilité des trois polyélectrolytes conduit à une valeur moyenne de $U_{0}$ égale à $6,5 \times 10^{-4} \mathrm{~cm}^{2} \cdot \mathrm{s}^{-1} \cdot \mathrm{V}^{-1}$. On observe également une pente comparable pour les 3 échantillons de l'ordre de $k \simeq 2(\mathrm{M} / \mathrm{L})^{-1 / 2}$.

Dans cette interprétation, nous avons considéré que l'effet électrophorétique en $(1+x R)^{-1}$ était responsable de la variation de la mobilité avec le sel ajouté.

Une autre approche consiste à considérer l'évolution de la viscosité intrinsèque de la solution. Celle-ci varie suivant une loi du type $A+\frac{B}{C_{\mathrm{s}}^{+1 / 2}}$ [34] en négligeant la contribution de $C|Z| / 2 M$. Dans ces conditions également on prévoit que la mobilité varie linéairement $C_{\mathrm{s}}^{-1 / 2}$. La complexité des solutions de polyélectrolytes en présence de sel ajouté, qui modifie à la fois la charge apparente de la macromolécule et la viscosité du milieu, ne nous permet pas de conclure sans ambiguité sur les rôles respectifs de ces deux effets.

Pour les polyélectrolytes tels que $\mathrm{ChSO}_{4} \mathrm{Na}$ $(C=1,5 \mathrm{~g} / \mathrm{l})$, l'évolution de la mobilité avec la force ionique est décroissante en $C_{\mathrm{s}}^{+1 / 2}$ uniquement aux très faibles valeurs de $C_{\mathrm{s}}\left(10^{-3} \mathrm{M} / \mathrm{L}\right)$. En excès de sel, la mobilité tend vers une valeur constante bien que toutes les charges ne soient pas écrantées. L'expression donnée par Gorin (éq. (11)) pour une molécule en forme de bâtonnet rigide semble être mieux adaptée pour décrire cette évolution (Fig. 9). Aux forces ioniques élevées, les résultats expérimentaux sont supérieurs aux valeurs théoriques.

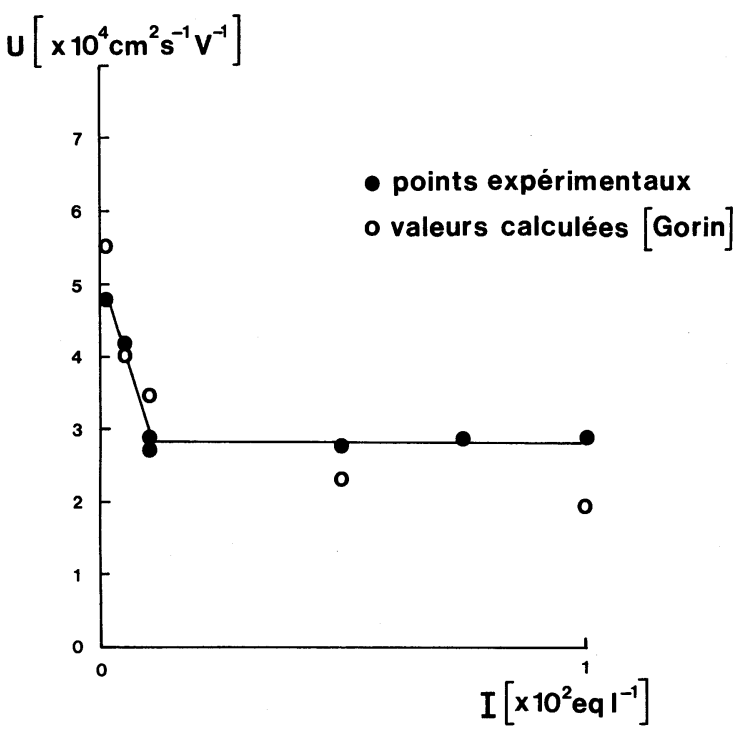

Fig. 9. - Variation de la mobilité électrophorétique du $\mathrm{ChSO}_{4} \mathrm{Na}$ avec l'addition d'électrolyte $\mathrm{NaCl}$.

[Evolution of electrophoretic mobility of $\mathrm{ChSO}_{4} \mathrm{Na}$ with the $\mathrm{NaCl}$ salt concentration.]

5.4. EVOLUTION DE LA MOBILITÉ ÉLECTRONIQUE AVEC LA CONCENTRATION EN POLYIONS SANS SEL AJOUTÉ. - Les domaines de concentrations étudiés pour ces différentes macromolécules sont définis par rapport à une concentration critique $C^{*}$. $\left(C^{*}=M / N V\right.$, $M$ étant la masse du polyion, $V$ le volume occupé par le polyion dans les conditions de l'expérience et $N$ le nombre d'Avogadro.)

- Pour la BSA $C^{*} \sim 70 \mathrm{~g} / \mathrm{l}$ et toutes les expériences sont faites en régime dilué.

- Pour le copolymère d'acide maléique de poids moléculaire compris entre $3 \times 10^{5}$ et $10^{6}$, les concentrations étudiées, sont toujours très supérieures à $C^{*}$ (régime semi-dilué).

- Pour le PMA et le $\mathrm{ChSO}_{4} \mathrm{Na}$, les concentrations critiques sont difficiles à définir précisément, du fait des changements de conformation. En absence de sel, les chaînes ont tendance à être totalement étirées et les concentrations critiques sont respectivement 
de l'ordre de $0,75 \mathrm{~g} / \mathrm{l}$ et $1,6 \mathrm{~g} / \mathrm{l}$. Notre domaine d'étude se situe entre 0,3 et $10 \mathrm{~g} / 1$ de sorte que nous considérons que l'essentiel des résultats obtenus est en régime semi-dilué $C \geqslant C^{*}$ et les modèles proposés dans ce régime sont discutés.

Deux types de variations expérimentales de $U$ en fonction de $C(\mathrm{~g} / \mathrm{l})$ sont observés. Dans le cas du BSA et du copolymère d'acide maléique et d'éthyl vinyl éther, la mobilité électrophorétique est sensiblement indépendante de $C$. Au contraire pour le PMA et le $\mathrm{ChSO}_{4} \mathrm{Na}$ nous observons une décroissance notable avec la concentration molaire et la loi de variation de $U$ est en $C_{\mathrm{M}}^{-1 / 4}$ pour $\mathrm{ChSO}_{4} \mathrm{Na}$ et $C_{\mathrm{M}}^{-1 / 6}$ pour le PMA (Fig. 10).

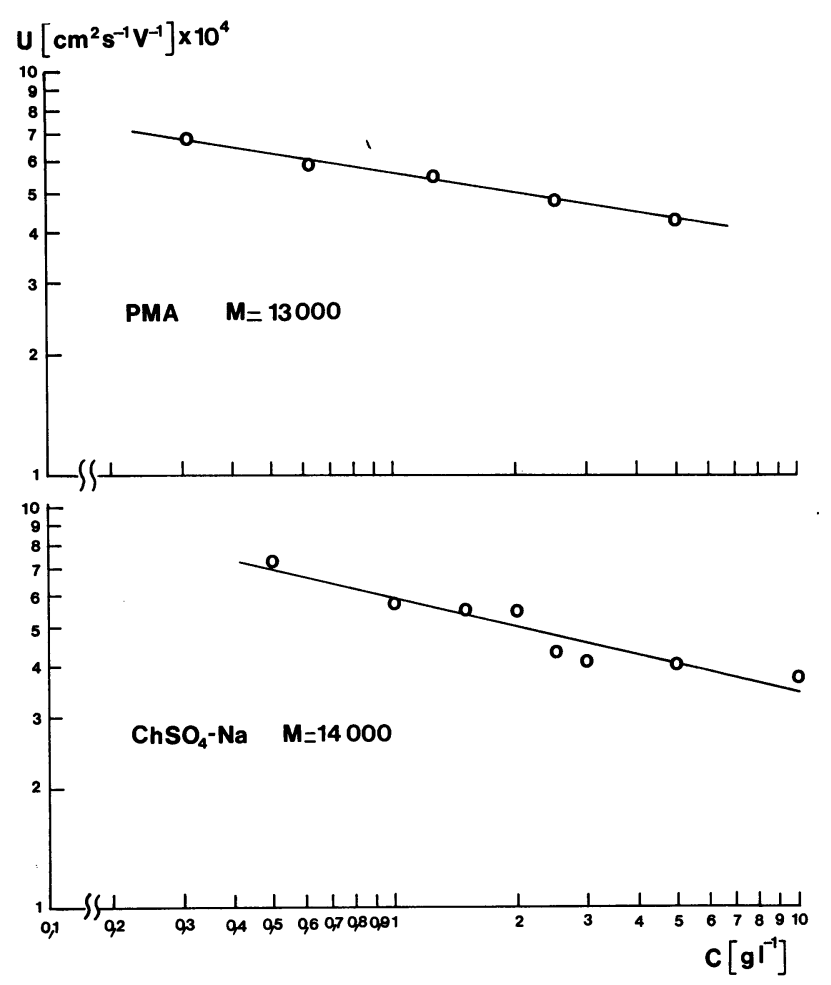

Fig. 10. - Variation de la mobilité électrophorétique du PMA et du $\mathrm{ChSO}_{4} \mathrm{Na}$ avec la concentration en polyions sans sel ajouté.

[Variation of electrophoretic mobility of PMA and $\mathrm{ChSO}_{4} \mathrm{Na}$ with the polyion concentration without salt.]

Considérons toujours l'expression de la mobilité en fonction de la charge, du coefficient de friction et de la force ionique (relation (10) avec $C_{\mathrm{s}}=0$ ).

1) Nous faisons l'hypothèse que $R$ et $\eta$ sont indépendants de $C$ en régime dilué.

Nous pouvons écrire dans la limite $x R<1$

avec

$$
U=U_{0}(1-K \sqrt{C})
$$

$$
K=3,3 \times 10^{7} R \sqrt{\frac{|Z|}{2 M}}
$$

Les valeurs théoriques de $K$ sont de 0,1 pour la BSA alors que $K_{\text {th }} \simeq 0$ pour le copolymère.

Les données expérimentales pour le copolymère (Tableau II de la réf. [27]) donnent bien une constance de la mobilité avec la concentration. Pour le BSA nous observons une très légère décroissance linéaire en $\sqrt{C}$ de $4,8 \times 10^{-4} \mathrm{~cm}^{2} \cdot \mathrm{s}^{-1} \cdot \mathrm{V}^{-1}$ à $5 \mathrm{~g} / 1$ jusqu'à $3,6 \times 10^{-4} \mathrm{~cm}^{2} \cdot \mathrm{s}^{-1} \cdot \mathrm{V}^{-1}$ pour $50 \mathrm{~g} / \mathrm{l}$. La pente $\left(K_{\text {exp }}\right)$ est de l'ordre de 0,05 . Les résultats expérimentaux semblent en accord avec le fait que $R$ et $\eta$ sont indépendants de la concentration et que l'effet électrophorétique est faible.

2) Plusieurs modèles sont proposés pour décrire la structure des solutions de polyélectrolytes en régime semi-dilué :

- le modèle de Lifson et Katchalsky [35] qui assimile la solution de polyélectrolytes à une succession de domaines dans lesquels les polyions sont des cylindres structurés parallèlement. La distance interpolyions décroît lorsque la concentration augmente, ce qui entraîne un accroissement des forces coulombiennes entre les polyions. Ceci justifie, a priori, la décroissance expérimentale de la mobilité ;

- le modèle isotrope de De Gennes [36, 37] qui considère les chaînes de polyélectrolytes comme une succession de blobs de taille $\xi$. Les effets électrostatiques sont importants à l'intérieur des blobs. Chaque chaîne du polyélectrolyte a un comportement idéal et son rayon globulaire varie avec la concentration. Dans cette hypothèse, la viscosité réduite de la solution est sensible à la concentration en polyions.

D'après ce modèle, $R$ varie en $C_{\mathrm{M}}^{-1 / 4} N^{1 / 2}(N$ est le degré de polymérisation et proportionnel au nombre de groupes ionisés, et $C_{\mathrm{M}}$ est la concentration molaire). La viscosité spécifique varie comme $f(N) C_{\mathrm{M}}^{1 / 2}$.

Des données expérimentales, dans le cas du PMA, déduites des mesures faites par diffusion de neutrons, indiquent que $R$ varie en $C_{\mathrm{M}}^{-1 / 4}$ [36]. Les résultats en viscosité sont bien décrits par la relation $[36,38]$ :

$$
\frac{\eta-\eta_{0}}{\eta_{0}} \sim N^{1 / 2} C_{\mathrm{M}}^{1 / 2} .
$$

En considérant l'expression de la mobilité, corrigée de l'effet électrophorétique, celle-ci peut se mettre sous la forme :

$$
U \simeq \frac{A C_{M}^{-1 / 4}}{1+\alpha C_{M}^{+1 / 4}} \text { avec } \alpha \simeq 1,6 \times 10^{7}|Z| M^{-1 / 2} .
$$

Pour le sulfate de chondroitine, le terme $\alpha C^{+1 / 4}$ est relativement petit, de sorte que $U$ varie théoriquement comme $C_{\mathrm{M}}^{-1 / 4}$. Cette évolution est vérifiée expérimentalement ce qui semble indiquer que son rayon globulaire doit varier avec $C$. Des études ultérieures en diffusion centrale des neutrons devraient confirmer ce résultat. 
Une théorie électrostatique récente due à T. Odijk [45] sur les solutions semi-diluées de polyélectrolytes considère une variation du rayon globulaire d'une chaîne en $C_{\mathrm{M}}^{-\beta}$ avec $\beta=3 / 16$ ou $5 / 16$ suivant différents domaines de concentration. (Dans le modèle isotrope $\beta=1 / 4$.) En considérant une même variation de la viscosité

$$
\frac{C_{M}^{-5 / 16}}{1+\alpha C_{M}^{5 / 16}} \text { ou } \frac{C_{M}^{-3 / 16}}{1+\alpha C_{M}^{+3 / 16}} \text {. }
$$

Les variations expérimentales des mobilités des 2 polyélectrolytes linéaires $\left(\mathrm{ChSO}_{4} \mathrm{Na}\right.$ en $C_{\mathrm{M}}^{-1 / 4}$ et PMA en $C_{\mathrm{M}}^{-1 / 6}$ ) sont en accord qualitatif avec ce modèle et ne permettent pas de choisir entre le modèle isotrope de De Gennes et le modèle électrostatique de T. Odijk.

5.5 EVOLUTION DE LA MOBILITÉ ÉLECTROPHORÉTIQUE AVEC LA MASSE DU POLYÉleCTROLYTE. - Trois polyélectrolytes ont été étudiés à différentes masses (PMA-ADN-copolymère d'acide maléique). Dans tous les cas, il semble que la valeur de la mobilité électrophorétique soit insensible à la masse totale du polyélectrolyte et surtout au rapport charge sur masse (Figs. 8 et 11). Cette observation a été mise en évidence par d'autres techniques d'électrophorèse $[39,40]$.

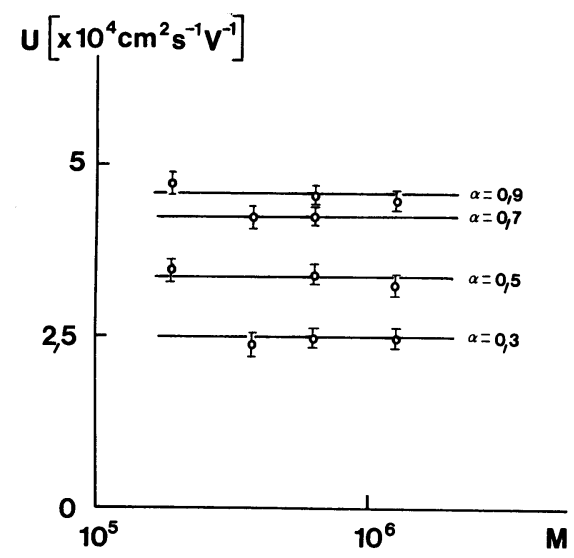

Fig. 11. - Evolution de la mobilité électrophorétique du copolymère d'acide maléique en fonction de la masse moléculaire et à différents degrés de neutralisation $\left(C_{\mathrm{s}}=0,05 \mathrm{M}\right.$ et $\left.C=0,5 \%\right)$.

[Dependence of electrophoretic mobility of copolymer of maleic acid on the molecular weight at various degrees of neutralization with $C_{\mathrm{s}}=0.05 \mathrm{M}$ and $C \simeq 0.5 \%$.]

Considérons toujours le modèle isotrope : le rayon varie en $N^{1 / 2} C_{\mathrm{M}}^{-1 / 4}$, alors que la relation entre la viscosité et le degré de polymérisation est définie $\operatorname{par} \eta \simeq f(N) C_{\mathrm{M}}^{1 / 2}$.

Dans l'expression générale (10) la mobilité varie avec le degré de polymérisation comme :

$$
\frac{N^{1 / 2}}{f(N)\left(1+\beta N^{1 / 2}\right)} \text {. }
$$

Pour le copolymère d'acide maléique [41] la variation de la viscosité intrinsèque suit la relation de Mark-Houwink $[\eta]=K M^{a}[34]$ avec $a \simeq 0,80$. Pour le PMA [38], le coefficient $a$ est de l'ordre de 0,50. D'une façon générale pour les macromolécules flexibles, $a$ varie entre 0,5 et 0,9 .

La relation établie par Katchalsky et Eisenberg [42] qui considère $a=0,5$ permettrait de justifier les données expérimentales, en négligeant le terme correctif d'effet électrophorétique en $\left(1+\beta N^{1 / 2}\right)^{-1}$.

Dans les évolutions de $U$ avec la concentration et la masse du polyion, nous avons considéré la viscosité de la solution dans l'expression du coefficient de friction. Celle-ci est en effet sensible aux conditions du milieu (concentration en contre-ions, en polyions et masse). Cette approche est justifiée qualitativement par les données expérimentales. Dans l'étude des propriétés hydrodynamiques des polymères neutres [46], il semble que l'on considère uniquement la viscosité du solvant. Dans ce cas, le coefficient de diffusion effectif :

$$
D(C) \sim \frac{k T}{6 \pi \eta_{0} \xi(c)} \text { varie en } C^{+3 / 4}
$$

( $\eta_{0}$ viscosité du solvant et $\xi(c):$ longueur de corrélation $\sim C^{-3 / 4}$ ).

Les résultats expérimentaux en diffusion de la lumière justifient cette approche sur les polymères neutres [47]. Nos résultats expérimentaux ne nous permettent pas de considérer la viscosité indépendante des conditions du milieu. Il semble que sous l'effet du champ électrique, le déplacement Doppler permet de déduire essentiellement la mobilité électrophorétique des chaînes individuelles pour les polyélectrolytes linéaires et de ce fait, est sensible au rayon globulaire $R(C)$. De même la viscosité doit être influencée par la densité de la double couche donc par la concentration des contre-ions ou simplement par les effets électrovisqueux [14].

5.6 EVOLUTION DE LA MOBILITÉ ÉLECTROPHORÉTIQUE AVEC LE PH DE LA SOLUTION. - L'étude de billes de polystyrène latex à différents $\mathrm{pH}$ montre une évolution de la mobilité qui a un maximum pour un pH compris entre 7 et 8 (Fig. 12). La charge apparente déduite de la loi de Nernst-Einstein varie entre 200 et 300. Par titration nous avons mesuré 280 groupes ionisables et il est donc tout à fait logique que pour un pH croissant entre 4 et 8 le nombre de groupements ionisés augmente. Ensuite, l'ionisation étant complète la charge apparente doit rester constante. La courbe expérimentale est en accord avec ces prévisions.

D'autres auteurs $[49,25]$ ont étudié les billes de polystyrène pour tester la technique de DQLCE et déterminer des mobilités électrophorétiques. Les valeurs obtenues sont difficilement comparables du fait que la charge surfacique des billes dépend essentiellement de leur préparation. 


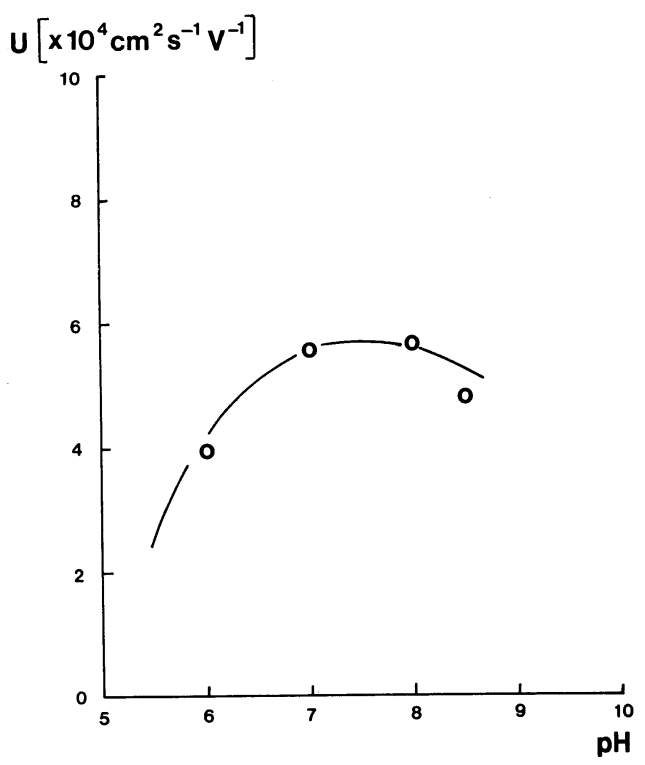

Fig. 12. - Variation de la mobilité électrophorétique d'une solution de billes de polystyrène latex avec le $\mathrm{pH}$ de la solution $\left(C_{\mathrm{s}}=10^{-3} \mathrm{M}\right.$ et $\left.C=0,005 \%\right)$.

[Evolution of electrophoretic mobility of latex polystyrene spheres solution with $\mathrm{pH}$ of the solution $\left(C_{\mathrm{s}}=10^{-3} \mathrm{M}, C=0.005 \%\right)$.]

6. Conclusion. - Cette technique de diffusion quasiélastique de la lumière sous champ électrique permet donc de suivre l'évolution des paramètres électrocinétiques des polyélectrolytes avec les conditions $d u$ milieu. Le fait d'étudier en veine liquide les macromolécules est fondamental pour la description des interactions électrostatiques. La rapidité et la précision des mesures sont très précieuses dans l'étude des composés biologiques, dont la structure peut se dégrader dans le temps.

Cette technique expérimentale nécessite encore des améliorations, d'une part, en perfectionnant la cellule électrophorétique et d'autre part, en adoptant une détection hétérodyne à faisceaux séparés. Enfin, un générateur séquenceur de tension stabilisée doit permettre de soumettre les macromolécules à un champ rigoureusement constant, quels que soient les phénomènes aux électrodes.

Les évolutions expérimentales des mobilités des différentes macromolécules avec les conditions du milieu (charge, sel ajouté, concentration, $\mathrm{pH}$ ) ont permis de préciser d'une façon phénoménologique quelques points intéressants dans la physico-chimie des polyélectrolytes.
L'approximation de Debye-Hückel dans l'expression de la mobilité électrophorétique en $(1+x R)^{-1}$ semble bien justifiée dans le cas de la BSA, où l'on maitrise la charge structurale avec la valeur du $\mathrm{pH}$. $\mathrm{La}$ charge apparente déduite des données expérimentales suivant la relation de Nernst-Einstein est en bon accord avec la valeur de cette charge apparente corrigée de l'effet électrophorétique,

$$
Z_{\text {app }}=Z(1+\chi R)^{-1} .
$$

La valeur de la charge apparente écrantée par les contre-ions semble également justifier l'évolution du coefficient de translation avec la force ionique du milieu [33, 43].

Pour les polyélectrolytes linéaires et flexibles, en régime dilué ou semi-dilué, les évolutions de la mobilité avec le sel ajouté et la concentration en polyion sont plus délicates à interpréter sans ambiguité. En effet le coefficient de friction et la charge apparente sont sensibles aux conditions du milieu et il semble que les effets de viscosité, et de taille de la macromolécule, puissent décrire à la fois la décroissance des mobilités avec $C_{\mathrm{s}}^{+1 / 2}$ et la variation en $C_{\mathrm{M}}^{-1 / 4}$ pour le $\mathrm{ChSO}_{4} \mathrm{Na}$. Toutefois, une justification qualitative des modèles isotrope et électrostatique des solutions de polyélectrolytes est proposée.

Un résultat très général a été mis en évidence. $\mathrm{La}$ mobilité électrophorétique est indépendante de la masse moléculaire et du degré de polymérisation. Des théories récentes de la mobilité électrophorétique sur des polyélectrolytes linéaires [44] confirment ces résultats.

Les théories électrostatiques de Stephen [33], reprises par Berne et Pecora [21], appliquées aux effets électrophorétiques sont à revoir à la lumière des résultats précédents. Des études expérimentales et théoriques de ces effets sont en cours pour mieux définir l'influence des interactions coulombiennes dans la solution en présence de champ extérieur [50].

Remerciements. - Les auteurs remercient P. Turq et $\mathrm{H}$. Magdelénat pour de très nombreuses discussions et $\mathrm{G}$. Weill et M. Moan pour la fourniture des échantillons d'ADN et de PMA. J. P. Meullenet et A. Schmitt ont participé directement aux études sur le copolymère d'acide maléique. Nous leur sommes très reconnaissants de bénéficier de leurs résultats.

\section{Bibliographie}

[1] Yeh, Y., Cummins, H. Z., Appl. Phys. Lett 4 (1964) 176

[2] Ware, B. R., Flygare, W. H., Chem. Phys. Lett. 12 (1971) 81.

[3] Mohan, R., Steiner, R. and Kaufmann, R., Anal. Biochem. 70 (1976) 506.

[4] Hartford, S. L. and Flygare, W. H., Macromolecules 8 (1975) 80.
[5] HaAs, D. D., Ware, B. R., Anal. Biochem. 70 (1976) 506.

[6] Uzgiris, E. E. and Kaplan, J. H., J. Colloid \& Interface Sci. 55 (1976) 148

[7] UzGiris, E. E., Opt. Commun. 6 (1972) 55.

[8] Siegel, D. P., et al., Biophys. 5 (1978) 624.

[9] Ware, B. R., Adv. Colloid \& Interface Sci. 4 (1974) 1. 
[10] Plygare, W. H., Ware, B. R. and Hartford, S. L., in Molecular Electrooptics, Part I (Marcel Dekker, N. Y.) 1976, p. 321.

[11] WARE, B. R., in Chemical and Biochemical Applications of Lasers, VII (Acad. Press, N. Y.) 1977, p. 199.

[12] Smith, B. A. and WARE, B. R., in Analytical and Chemical chemistry, V2 (Plenum Press, N. Y.) 1978, p. 29.

[13] UzGIRIs, E. E., Laser Doppler Electrophoresis à paraittre dans Prog. in Surf. Sci. (1980).

[14] BIER, M., Electrophoresis (Acad. Press) 1959.

[15] Henry, D. C., Proc. R. Soc. London A 133 (1931) 106.

[16] Abramson, H. A., Gorin, M. H., Moyer, L. S., Chem. Rev. 24 (1939) 345.

[17] Einstein, A., Anal. Phys. 17 (1905) 549.

[18] Magdelenat, H., TurQ, P., Tivant, P., Chemla, M., Menez, R., DrIFFord, M., J. Chem. Ed. 55 (1978) 12.

[19] Magdelenat, H., TurQ, P., Tivant, P., Chemla, M., Menez, R., DRIFFoRD, M., Biopolymers 18 (1979) 187.

[20] Chu, B., Laser light scattering (Acad. Press, N. Y.) 1974.

[21] Berne, B. J. and PeCora, R., Dynamic light scattering (Wiley, N. Y.) 1976.

[22] Menez, R., Thèse de Doctorat d'Etat, Orsay (1979).

[23] Menez, R., Arnaud, B. et Drifford, M., C. R. Hebd. Séan. Acad. Sci. Paris (1975) 280.

[24] HAAS, D. D., Electrophoretic light scattering of dilute proteines solutions $\mathrm{PhD}$ (1978) Harvard University, Cambridge Massachusetts.

[25] UzGIRIs, E. E., Rev. Sci. Instrum. 45 (1974) 74.

[26] Lemerle, J., Magdelenat, H., Lefebre, J., Bull. Soc. Chim. $56(1974) 753$.

[27] Meullenet, J. P., Schmitt, A., Drifford, M., J. Phys. Chem. 83 (1979) 1924.

[28] Pefferkorn, E., Dejardin, P., Varoqui, R., J. Colloid \& Interface Sci. (1978) C3-383.
[29] Manning, G. S., J. Chem. Phys. 46 (1967) 2324 ; 51 (1969) 924 et 3249.

[30] Preston, B. N., Snowden, Mck., Hougton, K. T., Biopolymers 11 (1972) 1645.

[31] Arnaud, B., Legre, J., Drifford, M., J. Chem. Phys. 71 (1974) 591.

[32] Doherty, P. and Benedex, G. B., J. Chem. Phys. 61 (1974) 5426.

[33] Stephen, M. J., J. Chem. Phys. 61 (1974) 1598.

[34] Pals, D. T. F., Hermans, J. J., Rec. Trav. Chem. 71 (1952) 56.

[35] Lifson, S. and Katchalsky, A., J. Polymers 13 (1954) 43.

[36] De Gennes, P. G., Pincus, P., Velasco, R. and Brochard, F., J. Physique 37 (1976) 1461.

[37] Pfeuty, P., J. Physique Colloq. 39 (1978) C6-149.

[38] Moan, M., Thèse de Doctorat d'Etat, Université de Bretagne Occidentale (1976) No d'ordre B 3933.

[39] Noda, I., Nagasawa, M. and Ota, M., J. Am. Chem. Soc. 86 (1964) 5075.

[40] Olivera, B. M., Baine, P. and Davidson, N., Biopolymers 2 (1964) 245 .

[41] Meullenet, J. P., Thèse de Doctorat d'Etat, Université de Strasbourg (1978).

[42] Katchalsky, A., Eisenberg, H., J. Polym. Sci. 6 (1951) 145.

[43] Tivant, P., TurQ, P., Drifford, M., Magdelenat, H., and MENEZ, R., à paraître dans Biopolymers.

[44] Wander Drift, W. P. J. T., De Keizer, A. and Overbeek. J. H. J. Colloid \& Interface Sci. 1 (1979) 71.

[45] OdIJK, T., Macromolecules 12 (1979) 688.

[46] De Gennes, P. G., Macromolécules 9 (1976) 587.

[47] Adam, M., Delsanti, M., Macromolecules 10 (1977) 1229.

[48] Constantino, I., et al. Biopolymers 2 (1964) 1.

[49] Yoshimuza, T., et al., Japan. J. Appl. Phys. 11 (1972) 1974.

[50] TuRQ, P., Tivant, P., Drifford, M., à paraître. 